\title{
Amiloidosis cardiaca: reporte de un caso
}

Enrique Ruiz-Mori 1,3, Leonor Ayala-Bustamante ${ }^{1}$, Luis Taxa-Rojas ${ }^{3, a}$, Cristian Pacheco-Román ${ }^{2}$, Javier Alarcón-Santos ${ }^{1}$, Jorge Burgos-Bustamante ${ }^{1,3}$

\section{RESUMEN}

La amiloidosis cardiaca es una entidad clínica poco frecuente y por tanto poco conocida. Su presentación, por ser una enfermedad infiltrativa debido al depósito extracelular del amiloide en la pared cardiaca, es la de una cardiomiopatía del tipo restrictivo, que si bien puede ser sistémica, es la afección cardiaca la que determina su mal pronóstico.

Se presenta el caso de un paciente de 38 años de edad, que fue hospitalizado por disnea y fatiga severa, y al que se diagnosticó mieloma múltiple IgA de cadenas ligeras lambda, estadio clínico IIIB, e inició tratamiento con ciclofosfamida, talidomida y dexametasona. El paciente abandonó el tratamiento y regresó luego de un año y 2 meses con la enfermedad avanzada y con presencia de amiloidosis. El paciente evoluciona tórpidamente y fallece.

Se realiza una revisión de la literatura médica sobre los tipos de amiloidosis, la fisiopatología, los métodos diagnósticos y el tratamiento.

Palabras clave: Amiloidosis; Cardiomiopatías; Insuficiencia cardiaca; Cardiomiopatía restrictiva (Fuente: DeCS BIREME).

\section{Cardiac amyloidosis: a case report}

\section{ABSTRACT}

Cardiac amyloidosis is a rare clinical entity and, therefore, almost unknown. It shows as a restrictive cardiomyopathy, since it is an infiltrative disease caused by the extracellular deposition of amyloids in the cardiac wall. Although it may be systemic, the heart condition determines its poor prognosis.

This is the case of a 38-year-old patient who was admitted for dyspnea and severe fatigue. He was diagnosed with IgA multiple myeloma, lambda light chains, clinical stage IIIB, and initiated treatment with cyclophosphamide, thalidomide and dexamethasone. The patient stopped the treatment, and returned after one year and 2 months with an advanced disease and presence of amyloidosis. The patient progressed torpidly and eventually died.

A review of the medical literature is performed concerning amyloidosis types, physiopathology, diagnostic methods and treatment.

Keywords: Amyloidosis; Cardiomyopathies; Heart failure; Restrictive cardiomyopathy (Source: MeSH NLM).

1. Instituto Nacional de Enfermedades Neoplásicas, Servicio de Cardiología. Lima, Perú.

2. Instituto Nacional de Enfermedades Neoplásicas, Departamento de Oncología. Lima, Perú.

3. Universidad de San Martín de Porres, Facultad de Medicina Humana. Lima, Perú.

a. Jefe del equipo funcional de Patología Quirúrgica, Investigador Concytec. 


\section{INTRODUCCIÓN}

La amiloidosis es una enfermedad poco frecuente, con una incidencia aproximada de 3 a 5 pacientes por millón al año, es más frecuente en hombres y la edad promedio de presentación es alrededor de los 65 años. Se estima una prevalencia en Estados Unidos de la amiloidosis AL de $30 \mathrm{a}$ 45 mil pacientes ${ }^{(1)}$.

La amiloidosis puede afectar múltiples órganos, los más frecuentes son el riñón (70 a $80 \%$ ), en segundo lugar, el corazón (50 a $60 \%$ ), también afecta al hígado (25\%), el tracto gastrointestinal, el sistema nervioso y tejidos blandos. En algunos casos de amiloidosis ( $5 \%$ de frecuencia) el corazón puede estar comprometido en forma aislada (2).

Existen cinco tipos de amiloidosis que comprometen al corazón: la amiloidosis primaria de cadena ligera (AL), la amiloidosis familiar, la amiloidosis sistémica senil, la amiloidosis atrial aislada y la amiloidosis sistémica secundaria ${ }^{(3,4)}$.

La amiloidosis AL es la forma más frecuente, las fibrillas están constituidas por cadenas ligeras tipo lambda o kappa (frecuencia 3 a 1) y se asocia a trastornos linfoproliferativos, como el mieloma múltiple, la macroglobulinemia de Waldenström y linfomas. La presencia de mieloma múltiple y amiloidosis se presentan en forma conjunta en un 10 a $15 \%$ de casos. El compromiso sistémico en este tipo de amiloidosis se reporta hasta en un 70 \%. Afecta al riñón con mucha frecuencia (74\%), sin embargo, cuando el corazón está afectado ( $20 \%)$, marca un mal pronóstico. La supervivencia promedio de este tipo de amiloidosis es de 12 meses ( 8 a 15 meses).

La amiloidosis familiar (AF) es una enfermedad autosómica dominante con penetrancia variable, ocasionada por una mutación en el gen para la proteína plasmática transtirretina (mTTR) en el cromosoma 18. La transtirretina es una proteína sintetizada en el hígado que se encarga de transportar el retinol y la tiroxina, así como también participa en los procesos de regeneración neuronal. Este tipo de amiloidosis afecta al sistema nervioso y al corazón, y sus manifestaciones más frecuentes son los trastornos neuro sensoriales y del sistema autonómico (diarrea, incontinencia) ${ }^{(5)}$.

La amiloidosis sistémica senil se presenta casi siempre en varones mayores de 65 años, la transtirretina natural o nativa (WTTR) se deposita en el corazón lo cual determina los síntomas de insuficiencia cardiaca congestiva. El amiloide se ubica también en el tracto gastrointestinal, lengua, glándulas endocrinas, entre otros órganos. La supervivencia es de aproximadamente 6 años ${ }^{(6)}$.

La amiloidosis atrial aislada no afecta otro órgano; el péptido natriurético atrial es la proteína precursora del amiloide. Se presenta en mujeres mayores de 80 años, y ocasiona alteraciones en la conducción eléctrica de la aurícula y los pacientes presentan fibrilación auricular.

En la amiloidosis sistémica secundaria las fibrillas se forman a base de la proteína sérica $A$. Se asocia a estados inflamatorios crónicos como tuberculosis, artritis reumatoidea; o a neoplasias como la enfermedad de Hodgkin e hipernefroma. La afección renal determina la evolución de la enfermedad y es raro el compromiso cardiaco (2 a $5 \%$ ).

El amiloide (depósito proteico insoluble que adopta una estructura de "hoja plegada beta" dispuestas en forma antiparalela, que describe la forma de fibras) tiende a depositarse en el miocardio como agregados nodulares con diversas ramificaciones que rodean y aíslan a los miocitos y producen una cardiomiopatía restrictiva. En algunas ocasiones puede invadir las válvulas cardiacas, el endocardio y el pericardio, así mismo afectar el sistema de conducción eléctrico que favorece los trastornos del ritmo, como fibrilación auricular, bloqueo aurículo-ventricular, entre otros ${ }^{(7)}$.

Actualmente han sido documentadas 28 proteínas anormales causantes de la amiloidosis. El sistema ubiquitina-proteosoma juega un rol fundamental en mantener el recambio proteico en los diferentes órganos y, en la amiloidosis, se produciría una falla a este nivel ${ }^{(8)}$. La proteína fibrilar, al depositarse en el músculo cardiaco, desarrolla una fibrosis intersticial, lo que condiciona alteración de la función ventricular y provoca diversos grados de disfunción diastólica (por alteración de la relajación ventricular) que progresa a un patrón restrictivo con presiones telediastólicas incrementadas, hipertrofia ventricular, y lleva al paciente finalmente, a estadios terminales con deterioro de la función sistólica y muerte ${ }^{(9)}$.

\section{CASO CLÍNICO}

Varón de 38 años de edad, natural y procedente de Lambayeque, obrero de construcción, actualmente desempleado; es derivado a cardiología por medicina oncológica por disnea a moderados esfuerzos, disfonía, tos con expectoración verdosa en regular cantidad y edema de miembros inferiores desde hace 4 meses. Tolera el decúbito, niega palpitaciones o dolor de pecho.

Examen físico: Presión arterial 90/60 mmHg. Frecuencia cardiaca 78 latidos/minuto. Saturación 02: $95 \%$. Frecuencia respiratoria 28 por minuto.

Aparato cardiovascular: Leve ingurgitación yugular. Ruidos cardiacos rítmicos, regulares, no tercer ruido, no soplos. Pulsos periféricos simétricos. Edema de miembros inferiores: $+/ 4+$. Aparato respiratorio: murmullo vesicular presente en ambos campos pulmonares. 
Electrocardiograma (ECG): Complejos bajo voltaje en derivadas de miembros y en V5-V6. Trastornos difusos de la repolarización ventricular (aplanamiento de la onda T) (Figura 1B).

Ecocardiograma: Disfunción sistólica de ventrículo izquierdo severa. Fracción de eyección $27 \%$. Hipocinesia global. Hipertrofia concéntrica de ventrículo izquierdo (Figura 3B, 4B) (SIV 1,41cm PPVI 1,31 cm DVItd: 3,67 cm DVIts 3,17 $\mathrm{cm}$ ), tipo amiloide moteado granular (Figura 5A). Dilatación biauricular y de ventrículo derecho y trombo en aurícula derecha (Figura 2B, 5B). Derrame pericárdico leve (Figura $5 A)$. Disfunción diastólica tipo restrictivo de ventrículo izquierdo (Figura 6B). Regurgitación mitral y tricúspidea leve. Doppler tisular en anillo mitral septal, patrón restrictivo (Figura 7). Colapso disminuido de vena cava inferior.

TEM tórax: Engrosamiento pleuroapical bilateral de aspecto residual. Atelectasias laminares en ambas bases pulmonares. Lesión lítica en manubrio esternal.

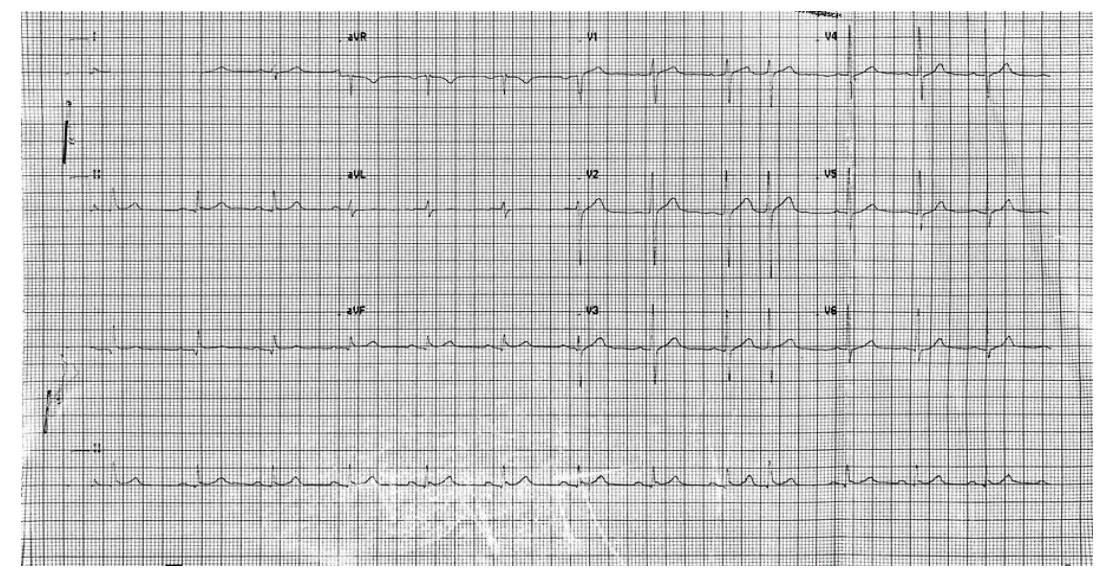

Figura 1A. ECG Basal: 24 de agosto 2016. FC: 79. Ritmo sinusal. Eje QRS 58 ${ }^{\circ}$. Intervalo PR: 179 ms. Complejo QRS:89 ms. QT: 339. QTc: 373 ms. Extrasístole supraventricular esporádica. Resto del trazo dentro de límites normales

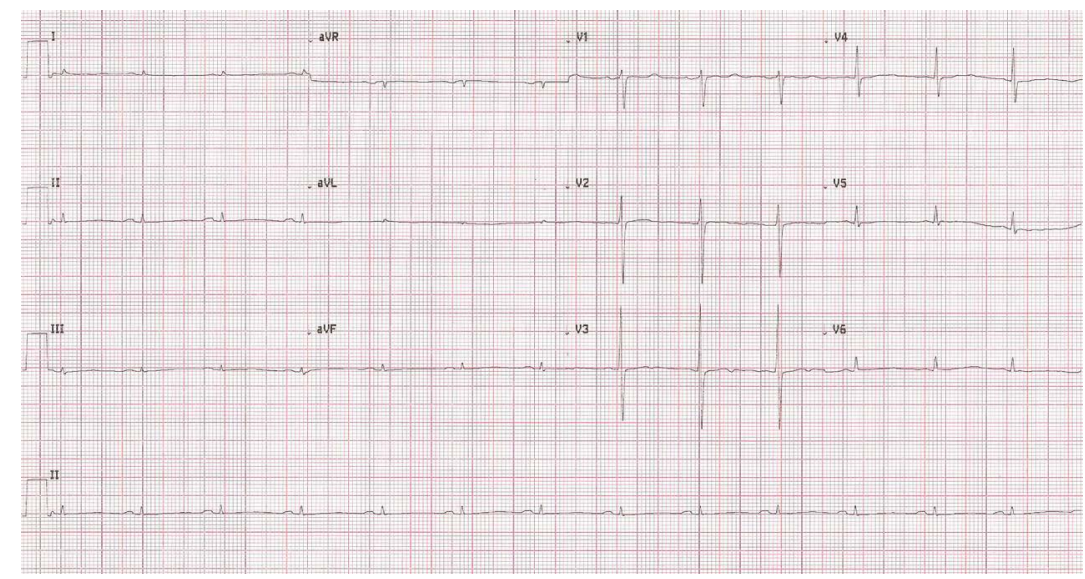

Figura 1B. ECG 14-4-2018: FC: 78 Ritmo sinusal eje QRS 63‥ Intervalo PR: 159 ms. Complejo QRS:94 ms. Amplitud del QRS en D1, D3 y aVL 0,1mV. QT: 397 QTc: 430 ms. Bajo voltaje de QRS en derivadas de extremidades. Anormalidad de la onda T difusa

Antecedentes: Paciente que ingresa al INEN el 23 de diciembre del 2014 con un tiempo de enfermedad de 2 meses caracterizado por disnea y fatiga severa al deambular, baja de peso (12 kg en 2 meses).
Estudio de médula ósea (30-12-2014): Compromiso medular por neoplasia de células plasmáticas de tipo mieloma de células plasmáticas (40\% de plasmocitos). Dosaje de cadenas ligeras IgA 22,8 ( $\mathrm{VN} 0,8-4,9)$. 
Diagnosticado el 31-12-2014 de mieloma múltiple IgA y cadenas ligeras lambda EC IIIB, recibió tratamiento con ciclofosfamida, talidomida y dexametasona.

El 11-2-2015 considerando una depuración de creatinina de $35 \mathrm{ml} / \mathrm{min}$, proteinuria 2,15 gr en 24 horas, creatinina de $225 \mathrm{mg} / \mathrm{dL}$, se concluye como enfermedad renal crónica secundario a nefropatía por mieloma. Tratamiento: losartán 25 mg/día.

Evaluación cardiovascular (24-8-16) previa a trasplante autólogo de médula ósea (TAMO): No antecedentes cardiovasculares de importancia, asintomático cardiovascular. Electrocardiograma: contracciones prematuras supraventriculares esporádicas (Figura 1A). Ecocardiograma: disfunción diastólica de ventrículo izquierdo (Figura 6A), fracción de eyección $61 \%$ (Figura 4A). Resto del examen dentro de límites normales (Figura 2A, 3A).
Se programa para TAMO el 30-8-16, pero el paciente abandona el tratamiento.

5 de octubre del 2017 retoma el tratamiento. 27 de marzo de 2018 cursa con disnea a medianos esfuerzos y disfagia. 28 de marzo de 2018: Biopsia de recto: amiloidosis colónica (Figura 8).

Evolución: Se instaura tratamiento con bortezonib, dexametasona, talidomida y calcio. Evolución tórpida que cursa con insuficiencia respiratoria aguda secundaria a insuficiencia cardiaca por amiloidosis cardiaca, enfermedad renal crónica reagudizada, anemia moderada, posteriormente cursa con sepsis punto de partida respiratorio, shock distributivo que no responde al tratamiento. Fallece el 24 de julio de 2018.
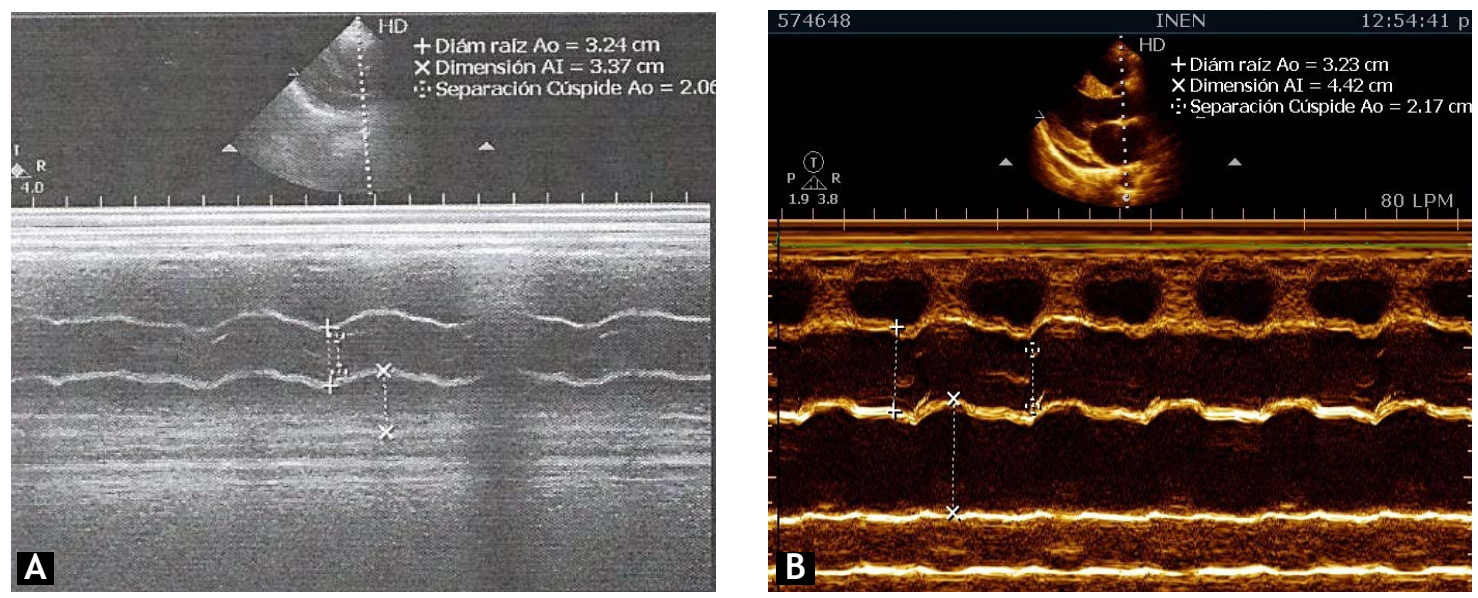

Figura 2. A. Eco basal: aurícula izquierda: $3,37 \mathrm{~cm}$ B. Amiloidosis: aurícula izquierda: 4,42 cm
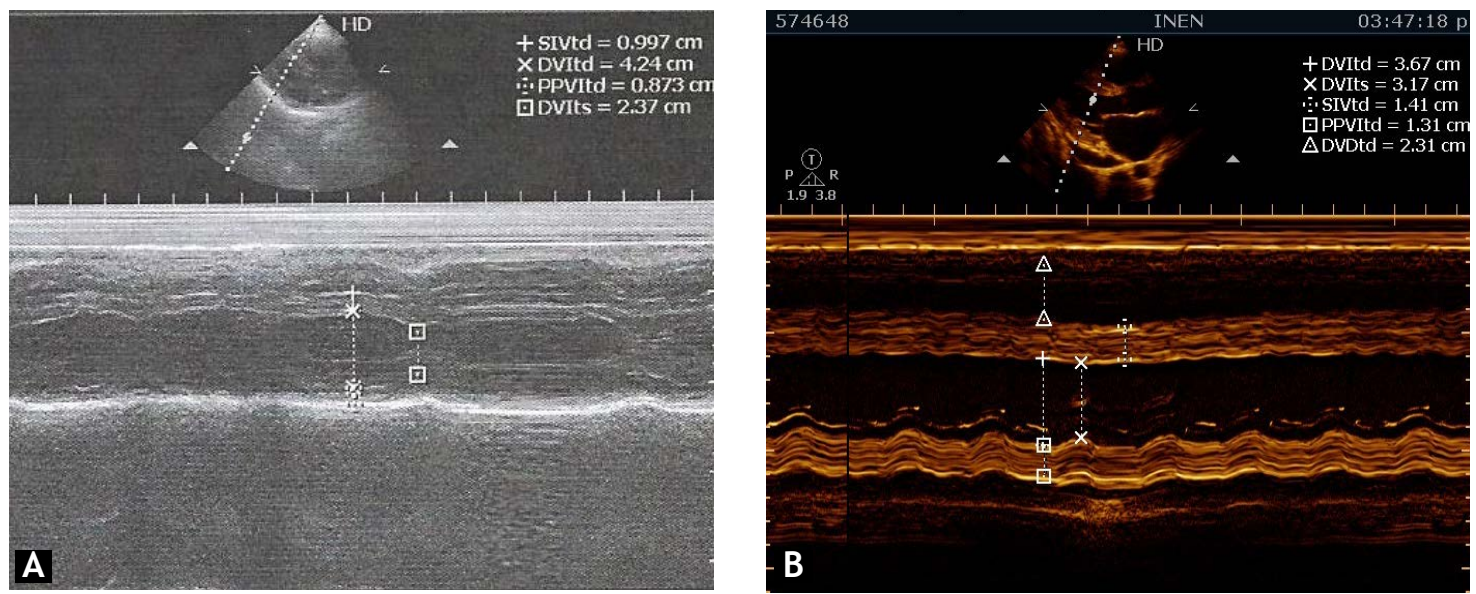

Figura 3. A. Eco basal: SIV: 0,99 cm. PPVI 0,87 cm. B. Amiloidosis: SIV 1,41 cm. PPVI 1,31 cm 

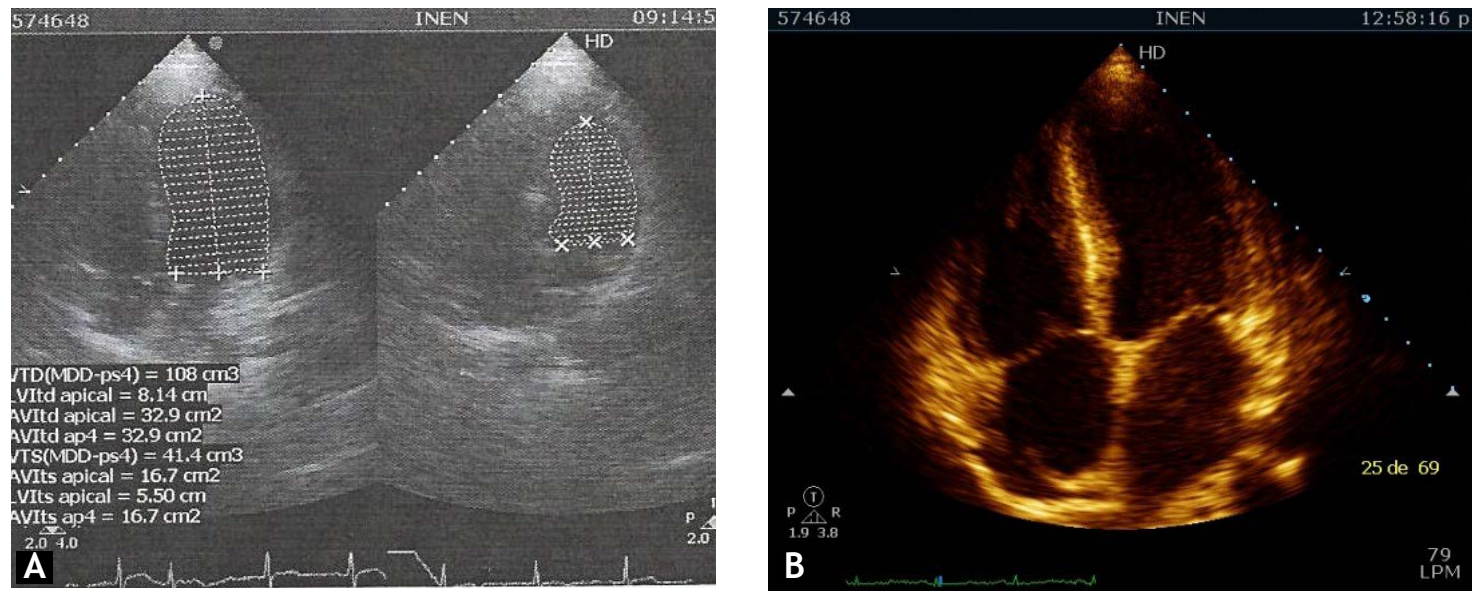

Figura 4. A. Basal: Fracción de eyección 61\% y paredes ventriculares normales. B. Amiloidosis: Vista apical (4 cámaras): engrosamiento de paredes ventriculares, dilatación biauricular
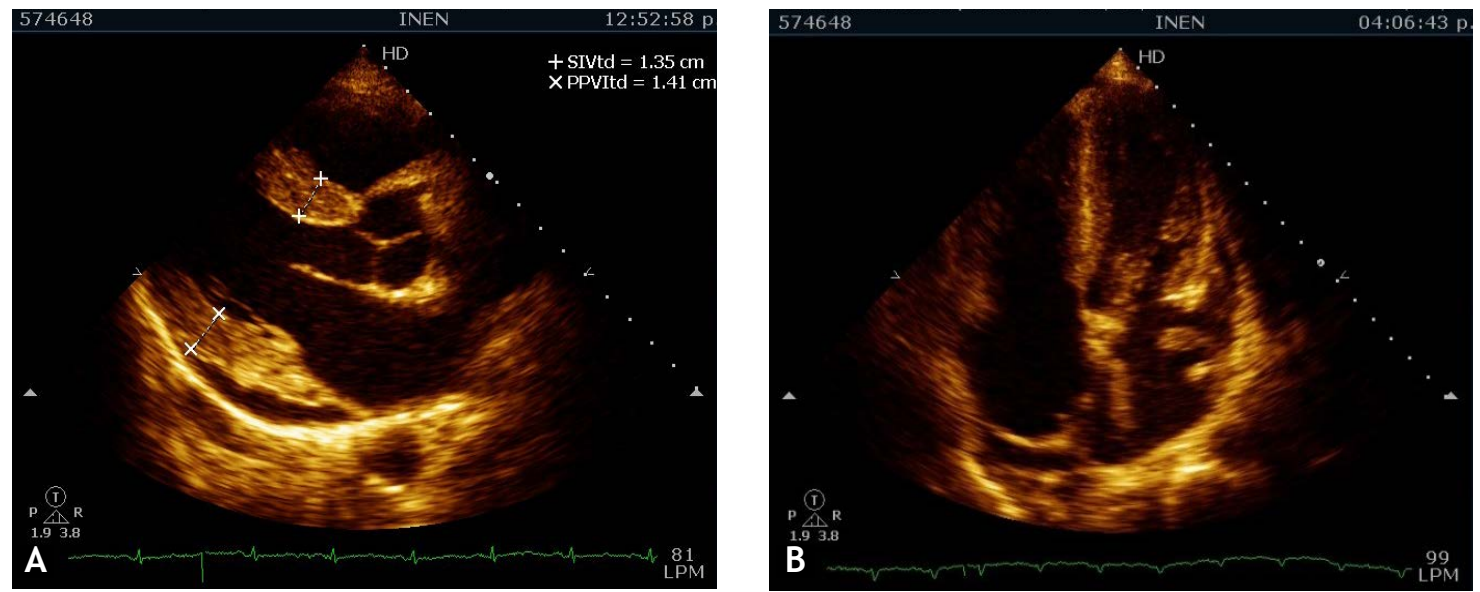

Figura 5. Amiloidosis: Vista paraesternal eje largo. Marcado engrosamiento miocárdico de ambos ventrículos con aspecto de patrón o textura granular. Mínimo derrame pericárdico. B. Amiloidosis: Vista apical (4 cámaras) donde se observa dilatación biauricular, engrosamiento del tabique interauricular y trombo en aurícula izquierda
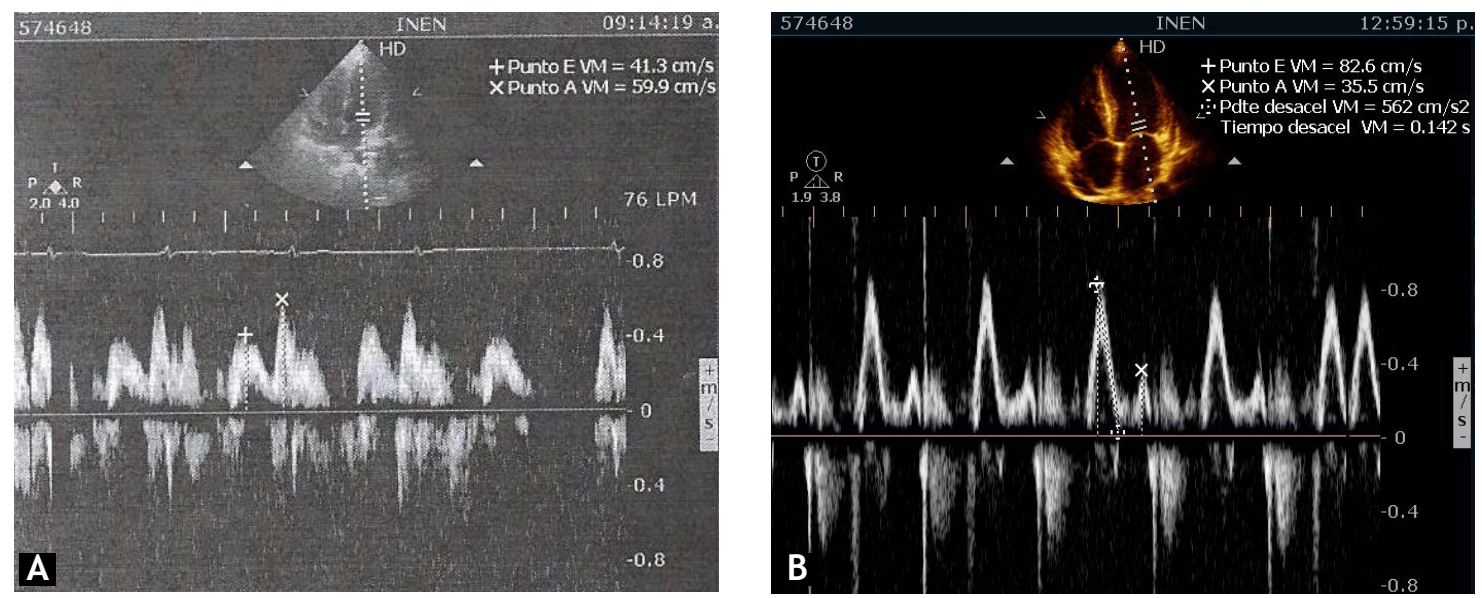

Figura 6. A. Eco basal: flujo mitral. Onda E: $41,3 \mathrm{~cm} / \mathrm{s}$. Onda A: $59,9 \mathrm{~cm} / \mathrm{s}$. B. Amiloidosis: Flujo mitral. Onda E $82,6 \mathrm{~cm} / \mathrm{s}$. Onda A 35,5 $\mathrm{cm} / \mathrm{s}$. E/A: 2,32. Tiempo de desaceleración: 0,142 s. Disfunción diastólica severa con patrón restrictivo 


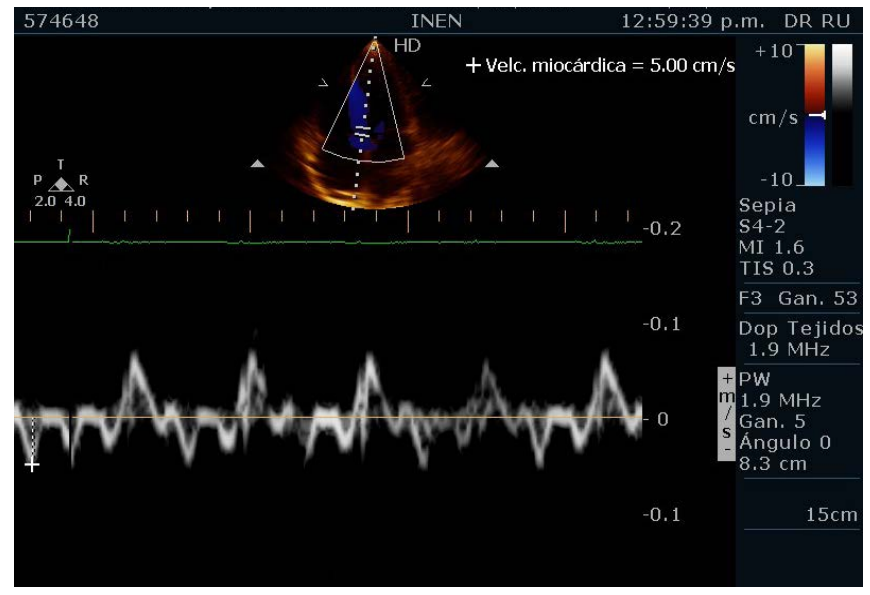

Figura 7. Amiloidosis: Eco doppler tisular en anillo mitral septal. Ondas E'y A'muy disminuidas. Relación onda E tisular/E mitral 0,06
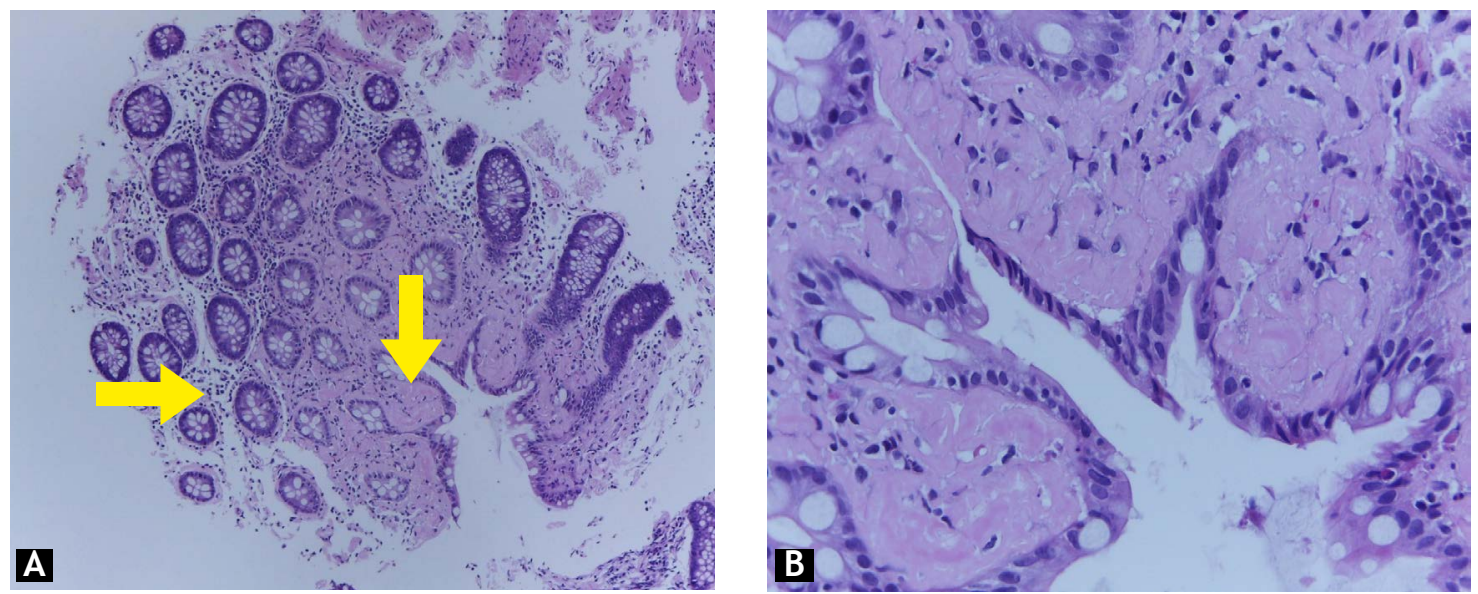

Figura 8. A. Se observa en este aumento (4X) la presencia de un material acidófilo en la lámina propia (flecha vertical) respecto a lo normal (flecha horizontal). B. A mayor aumento (20X) se ve con mejor detalle el depósito de material acidófilo acelular a nivel de lámina propia debajo del epitelio.

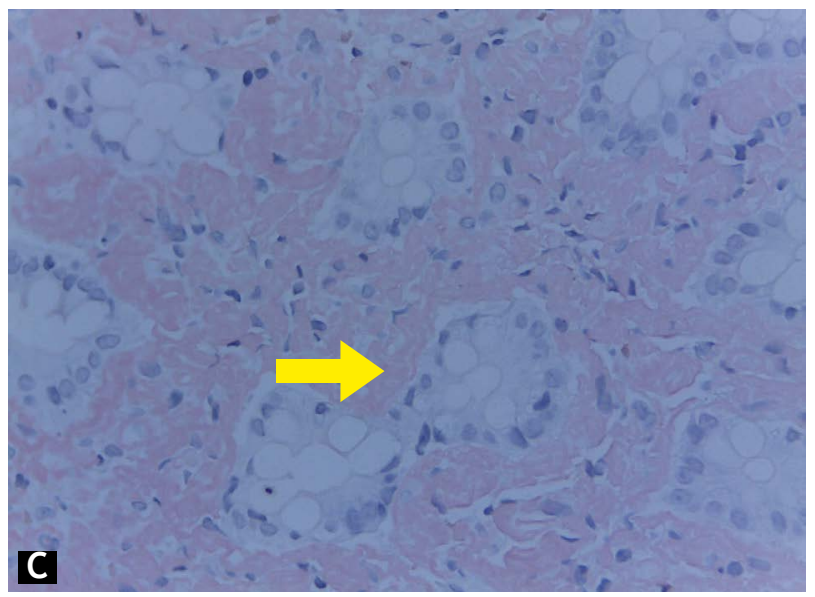

C. Esta coloración corresponde a rojo de Congo que permite resaltar el material acidófilo correspondiente a amiloide (flecha) 


\section{DISCUSIÓN}

El presente caso corresponde a una amiloidosis asociada a mieloma múltiple de cadenas ligeras, que es la forma más común de esta enfermedad ( 3 a 5 pacientes por millón por año) ${ }^{(10)}$, la cual tiene un pronóstico muy pobre, se estima que los pacientes sobreviven de 6 meses a 3 años, como ha sido este caso ( 3 años 7 meses).

Este paciente fue diagnosticado inicialmente de mieloma múltiple y recibió tratamiento por un lapso de 1 año y medio. No se pudo realizar el TAMO debido al abandono del plan de trabajo por parte del paciente, que perdió un año de tratamiento, por lo que la enfermedad progresó desfavorablemente.

Es importante destacar que el compromiso cardiaco en la amiloidosis es un marcador de mal pronóstico, por eso cuando se genera la insuficiencia cardiaca clínica y no se recibe tratamiento, el promedio de supervivencia es menor de 6 meses. Otro factor pronóstico es la cuantía de la cadena ligera circulante.

El cuadro clínico se caracteriza por la insuficiencia cardiaca, que se manifiesta en la mayoría de los casos con disnea, la cual es progresiva y se puede acompañar de edema periférico al que, en casos avanzados, se agrega ascitis. Otro síntoma característico es la presencia de una marcada astenia ${ }^{(11,12)}$.

En este caso el cuadro clínico fue disnea y astenia marcada, lo que motivó que el paciente acuda por atención médica. La literatura médica también describe que pueden presentarse episodios de síncope al esfuerzo, que es un signo de mal pronóstico (mortalidad en 3 meses). La presencia de palpitaciones en estos pacientes puede ser expresión de episodios de arritmias, sobre todo de fibrilación auricular (10 a $15 \%$ de frecuencia). Se reporta también angina de pecho, la cual puede ser expresión del depósito del amiloide en los vasos coronarios.

Las manifestaciones extracardiacas incluyen macroglosia (10 a $20 \%$ ), púrpura periorbitaria como expresión de la fragilidad capilar (15\%), síndrome de túnel carpiano, dolores neuropáticos, sequedad de boca, infiltración cutánea y depósitos periarticulares.

Al examen clínico se encuentra ingurgitación yugular, el pulso arterial puede ser normal o de menor amplitud. El primer y segundo ruidos cardiacos son normales y es poco frecuente la presencia de un tercer ruido. La presión arterial se torna baja como expresión del bajo gasto cardiaco y por la disfunción vasomotora periférica, producto de la neuropatía autonómica, también hay hipotensión ortostática, e incluso en sujetos hipertensos la presión tiende a disminuir. Se encuentra también hepatomegalia, ascitis y edema en miembros inferiores (13,14).

Los valores de troponina y de péptido natriurético cerebral (NT-proBNP) se encuentran elevados y son de importancia pronóstica para el paciente. La proteína ST2 soluble y la galectina-3 son nuevos biomarcadores que pueden ser de utilidad en estos casos.

Los cambios característicos en el electrocardiograma son la presencia de bajo voltaje (46-71\%), complejos QRS de amplitud menor de 0,5 $\mathrm{mV}$ en derivadas de miembros o menores de $1 \mathrm{mV}$ en precordiales, como se ha observado en este caso. También se describe el patrón de seudoinfarto por escasa progresión de la onda $\mathrm{R}$ en las derivadas precordiales de cara anterior en un $47 \%$ de casos. Cuando el amiloide compromete el sistema de conducción se puede apreciar bloqueos aurículo-ventriculares de primer grado $(21 \%)$, mientras que es menos frecuente el de segundo y tercer grado ( $3 \%)$, y, en algunos casos se puede observar bloqueo de rama derecha (9\%) o izquierda (5\%). La presencia de fibrilación y flutter auricular en amiloidosis es alrededor del $10 \%$. De acuerdo al tipo de amiloidosis, los cambios electrocardiográficos varían, así en la amiloidosis $\mathrm{AL}$ el 60 \% tienen complejos de bajo voltaje, mientras que en el senil son más frecuentes los bloqueos de rama $(40 \%){ }^{(15,16)}$.

En el ecocardiograma transtoráxico los cambios que caracterizan la amiloidosis son la hipertrofia concéntrica biventricular, pero en estadios iniciales puede sólo comprometer el septum; no hay dilatación de la cavidad; y se encuentra una fracción de eyección normal o ligeramente disminuida. El engrosamiento ventricular es causado por la presencia de un miocardio hiperecogénico que produce un aspecto típico granular o de vidrio esmerilado o moteado. También existe dilatación biauricular como consecuencia de la sobrecarga de presión en el llenado ventricular (volumen auricular izquierdo $>34 \mathrm{ml} / \mathrm{m} 2$ ), además puede existir el engrosamiento del tabique interauricular; es necesario descartar la presencia de un trombo en la cavidad auricular. El flujo transmitral muestra una onda E disminuida y una onda $A$ incrementada, disminuyendo la relación $\mathrm{E}: \mathrm{A}$, sin embargo, cuando la enfermedad progresa, la presión intraauricular se eleva lo que ocasiona un aumento de la velocidad inicial del llenado, y produce una seudonormalización de la relación E:A. En estadios finales lo característico es un patrón restrictivo con un tiempo de desaceleración de la onda $E$ reducido (menor de 160 mseg) y una onda A de velocidad baja (relación $E / A>1,5$ ). El doppler tisular muestra reducción en las velocidades diastólicas. El derrame pericárdico se encuentra en 40 a $60 \%$ de los pacientes, y es, generalmente, poco significativo.

En el strain longitudinal se puede encontrar que los segmentos basales y medios están disminuidos hasta 
en un $50 \%$, mientras que los segmentos apicales están conservados, lo que diferencia de los patrones hipertróficos. El strain rate puede detectar precozmente el deterioro de la función contráctil.

La resonancia magnética es de gran utilidad en los estadios avanzados de amiloidosis, pero al inicio su validez no es la misma. Lo característico de este examen en la amiloidosis es la captación difusa del gadolinio, que es marcado en la región subendocárdica en realce tardío. El patrón difuso es lo que permite diferenciar la amiloidosis de la enfermedad coronaria isquémica, donde el patrón es circunscrito a un territorio coronario. En algunos casos, se observa el realce parcheado o moteado, que puede ser localizado o transmural $(17,18)$. Este examen alcanza una sensibilidad de $80 \%$ y una especificidad de $94 \%{ }^{(4)}$. En este paciente no se pudo realizar este estudio.

El diagnóstico de la amiloidosis se realiza con la presencia del amiloide en los tejidos, para ello será necesario realizar la biopsia de grasa subcutánea abdominal (con una positividad del $80 \%$ de los casos), biopsia rectal (70\% de positividad), médula ósea (55\%) o la biopsia del órgano afectado. La biopsia endomiocárdica tiene una sensibilidad del $100 \%$, sin embargo, no es un procedimiento que se realice con frecuencia. Luego la muestra obtenida se tiñe con rojo Congo y bajo luz polarizada lo característico es observar una birrefringencia verde manzana. Para determinar el tipo de amiloide se realizará estudios de inmunohistoquímica. En este paciente la biopsia rectal brindó el diagnóstico ${ }^{(4)}$.

El pronóstico del paciente es desfavorable, generalmente fallecen por insuficiencia cardiaca o insuficiencia renal, aunque una arritmia puede ser el desencadenante de una muerte súbita por disociación electro-mecánica ${ }^{(19)}$.

El tratamiento de la amiloidosis cardiaca debe estar dirigido a dos objetivos, el primero es el manejo de la afección cardiaca y en segundo lugar de la enfermedad productora de la proteína amiloidótica. En relación a la terapéutica de la insuficiencia cardiaca está basada en el uso de diuréticos de asa, que en muchos casos, cuando hay hipoalbuminemia por el síndrome nefrótico, obliga a utilizar dosis altas; por ello siempre es necesario en estos pacientes el control del peso, balance hídrico permanente y restricción de la ingesta de sal. Últimamente, se está utilizando el biomarcador NT-proBNP como objetivo de tratamiento, buscando reducir en un $30 \%$ o $300 \mathrm{ng} / \mathrm{ml}$ de la cifra inicial ${ }^{(20)}$. Este paciente recibió tratamiento con diuréticos para el manejo de los signos y síntomas. También se puede utilizar en forma conjunta con un antagonista de la aldosterona como la espironolactona. El uso de betabloqueadores no es muy recomendado ya que la neuropatía autonómica asociada puede producir hipotensión y bradicardia. Así mismo, los inhibidores de la enzima convertidora de angiotensina (ECA) o los bloqueadores de los receptores de angiotensina (BRAT) no son muy recomendados por su pobre tolerancia, debido a que podrían generar hipotensión; si se decide usarlos, debe hacerse con mucho cuidado y a dosis muy bajas. Los antagonistas del calcio y la digoxina se pueden unir a los depósitos de amiloide, empeorando el cuadro clínico, lo que dificulta su uso. Como antiarrítmico se puede utilizar la amiodarona que es bien tolerada, sobre todo para el control de la frecuencia cardiaca en fibrilación auricular; y la anticoagulación es una alternativa en múltiples casos al considerar el compromiso auricular en esta enfermedad, la formación de trombos intraauriculares, como en este paciente, (Figura 5B) o por la presencia de fibrilación auricular ${ }^{(3,7,12)}$.

Para la amiloidosis AL, el tratamiento es el mismo que para el mieloma múltiple, ya que el origen de ambas afecciones está en las células plasmáticas anormales. El objetivo del tratamiento será erradicar la proteína precursora de fibrina, al suprimir la producción de cadenas ligeras tan rápido como sea posible. Los esquemas de quimioterapia recomendados son melfalan - dexametasona (evidencia Ila-B), vincristina - adriamicina - dexametasona (IIb-B), dosis alta de melfalan-transplante autólogo de células madre (Ila-B), talidomida-dexametasona (Ila-B), melfalandexametasona-bortezomib o ciclofosfamida-bortezomibdexametasona (Ila), y sobre todo en aquellos pacientes refractarios o con recaída de enfermedad lenalidomidadexametasona o pomalidomida-dexametasona (Ila) ${ }^{(10,21)}$.

\section{REFERENCIAS BIBLIOGRÁFICAS}

1. Quock TP, Yan T, Chang E, Guthrie S, Broder MS. Epidemiology of AL amyloidosis: a real-world study using US claims data. Blood Adv. 2018;2(10):1046-1053.

2. Cibeira T, Bladé J. Amiloidosis primaria: diagnóstico, pronóstico y tratamiento. Hematológica. 2011; 96 (1):13-18.

3. García-Pavía P, Tomé-Esteban M, Rapezzi C Amiloidosis. También una enfermedad del corazón. Rev Esp Cardiol. 2011;64:797-808.

4. Bhogal S, Ladia V, Sitwala P, Cook E, Bajaj K, Ramu V, Lavie CJ, Paul TK. Cardiac Amyloidosis: An Updated Review With Emphasis on Diagnosis and Future Directions. Curr Probl Cardiol. 2018;43(1):10-34.

5. Ruberg FL, Berk JL. Tranthyrerin cardiac amyloidosis. Circulation. 2012; 126 (10): 1286-1300.

6. Harrison C, Harrison D. Amiloidosis senil. Acta Med Grupo Angeles. 2018;16(1):58-62.

7. Fikrle M, Palecek T, Kuchynka P, Nemecek E, Bauerova L, Straub J, Rysava R. Cardiac amyloidosis: A comprehensive review. Cor et Vasa. 2013:55;E60-E75.

8. Rodríguez MA, Méndez MA. El Proteosoma, sus Inhibidores y Toxicidad Cardíaca. Rev Clín Esc Med. 2018;8(2):11-21.

9. Navarro-Ulloa OD, Conde-Cardona G, Gamero-Tafur JA, Ibarra-Jiménez G. Miocardiopatía restrictiva por amiloidosis. Rev Colomb Cardiol. 2016;23(6):525.e1---525.e5

10. Gertz M. Immunoglobulin light chain amyloidosis diagnosis 
and treatment algorithm 2018. Blood Cancer J. 2018;8(5):44.

11. Falk RH, Alexander KM, Liao R, Dorbala S. AL (Light-Chain) cardiac amyloidosis: a review of diagnosis and therapy. J Am Coll Cardiol. 2016;68 (12):1323-41.

12. Siddiqi OK, Ruberg FL. Cardiac amyloidosis: An update on pathophysiology, diagnosis and treatment. Trends Cardiovasc Med. 2018;28(1):10-21.

13. Duque $M$, Velásquez J, Marín J, Aristizábal J, Astudillo V, Medina L. Amiloidosis cardiaca. Rev Colomb Cardiol. 2009;16:118-127.

14. Núñez LE, Chacón S. Amiloidosis cardiaca: presentación de un caso y revisión de la literatuta. Rev Comb Cardiol. 2012;19(1):37-41.

15. Cyrille NB, Goldsmith J, Alvarez J, Maurer MS. Prevalence and prognostic significance of low QRS voltage among the three main types of cardiac amyloidosis. Am J Cardiol 2014;114:1089-93.

16. Sayago-Silva I. Amiloidosis AL Cardiaca Avanzada: Análisis de estrategias diagnósticas y Terapéuticas. [master's thesis] Madrid: Universidad Autónoma de Madrid; 2017. 128p.

17. Bhatti S, Watts E, Syed F, Vallurupalli S, Pandey T, Jambekar $\mathrm{K}$, et al. Clinical and prognostic utility of cardiovascular magnetic resonance imaging in myeloma patients with suspected cardiac amyloidosis. Eur Heart J Cardiovasc Imaging, 2016;17:970-977.

18. Donnelly J, Hanna M. Cardiac amyloidosis: An update on diagnosis and treatment. Cleve Clin J Med. 2017;84(3):12-26.

19. Cortes M, Battioni L, Sciancalepore A, Costabel J. Amiloidosis cardiaca simulando una miocardiopatía hipertrófica. Rev Fed Arg Cardiol. 2015;44(4):229-232.

20. Merlini G, Lousada I, Ando Y, et al. Rationale, application and clinical qualification for NT-proBNP as a surrogate end point in pivotal clinical trials in patients with $\mathrm{AL}$ amyloidosis. Leukemia. 2016;30(10):1979-1986.

21. Shameem Mahmood S, Palladini G, Sanchorawala V, Wechalekar A. Update on treatment of light chain amyloidosis. Haematologica. 2014; 99 (2):209-221.

Fuentes de financiamiento:

Este artículo ha sido financiado por los autores.

Conflictos de interés:

Los autores declaran no tener ningún conflicto de interés.

\section{Correspondencia:}

Enrique Ruiz Mori

Dirección: Av. Angamos 2520, Lima 34. Perú.

Teléfono: (511)998788580

Correo electrónico: cruizm@usmp.pe

Recibido: 18 de setiembre de 2018 Evaluado: 20 de setiembre de 2018 Aprobado: 09 de octubre de 2018

(c) La revista. Publicado por Universidad de San Martín de Porres, Perú. (cc) bajo términos de Licencia Creative Commons Atribución 4.0 Internacional. (http://creativecommons.org/licenses/by/4.0/)

\section{ORCID iDs}

Enrique Ruiz Mori

Leonor Ayala Bustamante

Luis Taxa Rojas

Cristian Pacheco-Román

Javier Alarcón-Santos

Jorge Burgos-Bustamante https: / / orcid.org/0000-0003-1248-5482 https: //orcid.org/0000-0002-1445-3268 https://orcid.org/0000-0002-0914-9149 https://orcid.org/0000-0003-2359-5126 https://orcid.org/0000-0003-4131-8333 https://orcid.org/0000-0001-8848-7586 